\title{
Migraine-associated Takotsubo cardiomyopathy
}

\author{
Francis Desmeules ${ }^{1} \mathbb{D} \cdot$ Stéphane Côté ${ }^{2}$
}

Received: 1 September 2021 / Accepted: 20 December 2021 / Published online: 5 January 2022

(C) The Author(s), under exclusive licence to Canadian Association of Emergency Physicians (CAEP)/ Association Canadienne de Médecine d'Urgence (ACMU) 2022

Keywords Migraine $\cdot$ Takotsubo $\cdot$ Chest pain

\section{Introduction}

Chest pain is the second most frequent cause of emergency department (ED) visits in Canada. In addition to the traditional risk factors for cardiovascular diseases, there is accumulating evidence suggesting that migraine is associated with life-threatening cardiovascular and cerebrovascular events such as myocardial infarction and stroke [1]. With an estimated $8.3 \%$ of Canadians diagnosed with migraine according to Statistics Canada, clinicians must be careful when evaluating chest pain in this population. Furthermore, migraine could also trigger Takotsubo cardiomyopathy, a stress-induced non-ischemic cardiomyopathy [2, 3]. Other acute neurological stressors including subarachnoid hemorrhage, intracerebral bleeding, epilepsy, traumatic brain injury, encephalitis, posterior reversible encephalopathy syndrome and stroke have been established as potential causal factors [4]. Our objective is to present an unusual case of Takotsubo cardiomyopathy precipitated by migraine, a disease that could by initially unrecognized due to its atypical presentation.

\section{Clinical presentation}

A 41-year-old woman presented to the ED with an history of unilateral intense throbbing 10-h headache, attenuated by a single $37.5+325 \mathrm{mg}$ dose of Tramadol-Acetaminophen,

Francis Desmeules

francis.desmeules.2@ulaval.ca

1 Département de Médecine Familiale Et Médecine d'Urgence, Faculté de Médecine, Université Laval, Quebec, QC, Canada

2 Laval University Emergency Department, 1050, avenue de la Médecine, Local 4617, Québec, Québec G1V 0A6, Canada compatible with her previous migraine headaches. Interview revealed that $3 \mathrm{~h}$ after the headache onset, as she was resting, the patient experienced right arm mild pain that radiated to her neck and back along with chest discomfort for $4 \mathrm{~h}$. She disclosed multiple similar episodes in the last 3 years, but did not seek medical care. The remaining symptoms at evaluation were the right arm pain and the headache. Her past medical history included Raynaud's syndrome, high blood pressure, fibromyalgia, irritable bowel syndrome and Arnold's neuralgia. She had no personal nor family history of cardiovascular diseases but had a past smoking history of 27 pack-years. Her daily medication included Nifedipine, Topiramate, Venlafaxine and Mirtazapine. She denied other physical nor psychological stressors. On examination, her blood pressure was 150/106 with a heart rate of 79 beats per minute. She had a saturation level of $100 \%$ in room air, respiratory rate of 16 breaths per minute, normal temperature and blood glucose level. The physical exam, including a neurological and cardiovascular exam, was noncontributory. The migraine headache was treated with metoclopramide $10 \mathrm{mg}$ and diphenhydramine $25 \mathrm{mg}$ intravenously. Since the arm pain was not explained by history and not reproduced by physical exam, troponin and electrocardiogram were performed. Point-of-care ultrasound showed no pericardial effusion.

The initial electrocardiogram showed non-specific $\mathrm{T}$-wave flattening in V1-V3. Troponin I was at $983 \mathrm{ng} / \mathrm{L}$ (normal $<54$ ). She was presumed to have an acute coronary syndrome since the pulmonary embolism ruleout criteria rule was negative and the chest radiograph showed a normal cardiomediastinal contour. Aspirin $320 \mathrm{mg}$, ticagrelor $180 \mathrm{mg}$ and fondaparinux $2.5 \mathrm{mg}$ were administered and a cardiology referral was made. Transthoracic echocardiography performed by a cardiologist $17 \mathrm{~h}$ after ED presentation demonstrated a slightly impaired left ventricle systolic function estimated at $45-50 \%$ with anteroapical, inferoapical and apical septal 
akinesia, compatible with acute myocardial infarction of the interventricular artery curving around apex or Takotsubo cardiomyopathy. Her coronary arteries were unremarkable on coronary angiography, and ventriculography showed a left ventricle ejection fraction of $51 \%$. Therefore, obstructive coronary disease was unlikely.

Her symptoms improved within a day of hospitalization, and she experienced no migraine relapse. Serial electrocardiograms were performed during her hospital stay, showing progressive deep T-wave inversions in V1-V6. She was discharged from the hospital after 3 days with aspirin and perindopril-indapamide.

Cardiac magnetic resonance imaging was performed 3 weeks after initial presentation and showed no myocardial injuries. Other causes of myocardial infarction with nonobstructive coronary arteries, such as coronary vasospasm, a presentation also linked to migraine, were therefore unlikely. Furthermore, the anomaly previously seen on echocardiography were resolved. One month after, the patient underwent a repeat transthoracic echocardiography that proved normal, with a normal left ventricle contractility and ejection fraction of $69 \%$. On this subsequent visit, electrocardiogram showed $\mathrm{T}$-wave inversions in V1-V4 (Fig. 1).
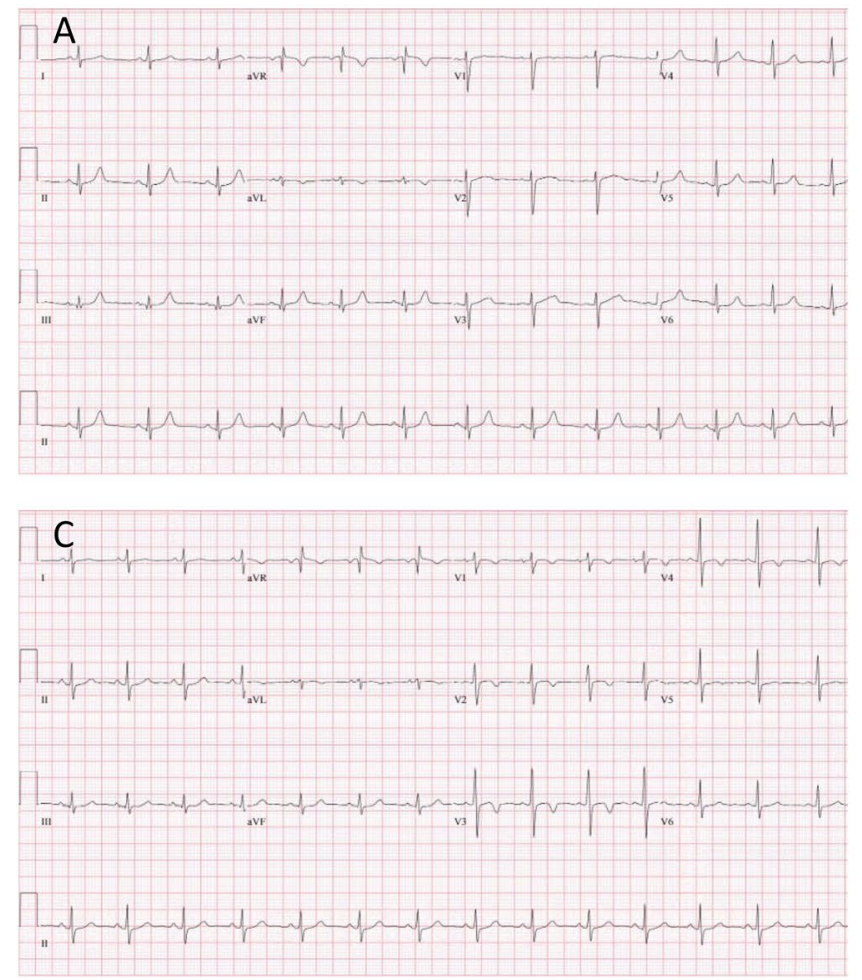

\section{Discussion}

We report a case of Takotsubo cardiomyopathy triggered by migraine in a 41-year-old woman, highlighting the diagnostic challenge of chest pain syndrome in premenopausal women. Multiple forms of Takotsubo cardiomyopathy have been described, with chest pain and dyspnea being the two predominant symptoms, mimicking acute coronary syndrome [4]. No randomized trial defined its optimal treatment, but cardiologists generally include beta-blockers and angiotensin-converting enzyme drugs to treat the resulting heart failure [4].

Many central nervous diseases are potential triggers, the more frequently associated being subarachnoid hemorrhage and seizures [4]. To our knowledge, only two case reports showed similar migraine associated Takotsubo cardiomyopathy and one case-control study on 25 patients with Takotsubo cardiomyopathy showed higher prevalence of migraine in this cohort compared to the age-matched control groups $[2,3,6]$.

Scientific literature has shown that almost $90 \%$ of Takotsubo cardiomyopathy were reported in female patients [4]. Unfortunately, there is a significant knowledge gap on the sex-related risk factors and causes of this condition, making its diagnosis particularly challenging. Among women

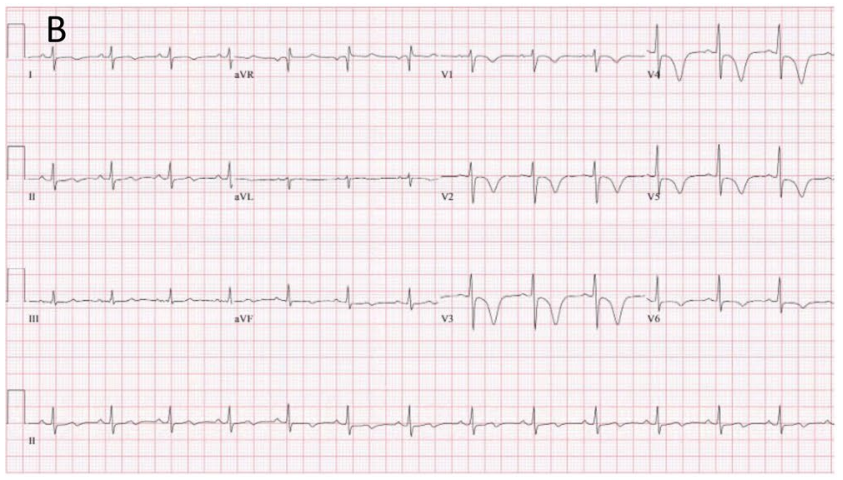

D

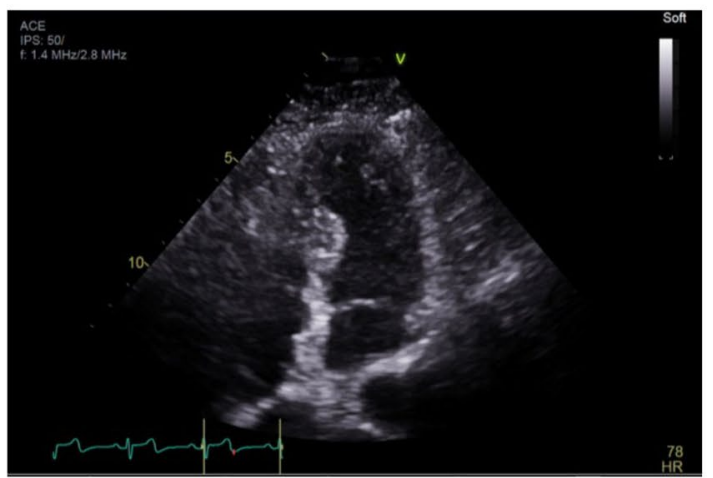

Fig. 1 Electrocardiogram at time of arrival (A), 72-h post arrival (B) and at 1-month follow-up (C). Four chambers view, echocardiography, demonstrating apical septal akinesia at the end of ventricular systole (D) 
with Takotsubo cardiomyopathy, small retrospective studies demonstrated a higher prevalence of migraine, and other vascular diseases such as Raynaud phenomenon [6]. Our patient suffered from both entities reinforcing this association. Furthermore, stress could also play a key role in this presentation since it could potentially trigger both migraine and Takotsubo cardiomyopathy but evidence suggests that the catecholamine excess alone cannot explain the latter, and patients are likely to have unique characteristics predisposing them to this condition [6].

Evidence is accumulating demonstrating the association between migraine and different cardiovascular and cerebrovascular diseases [1]. Such association is believed to be caused by the generalized endothelial dysfunction, cerebral hypoperfusion, higher prevalence of patent foramen ovale, hypercoagulable state and chronic nonsteroidal anti-inflammatory drugs use in migraineurs [1].

\section{Conclusion}

This case report shows that migraines could precipitate Takotsubo cardiomyopathy, through its vascular dysfunction pathway and/or as a direct trigger. Clinicians should remain vigilant of this association when assessing patients presenting with chest pain. Nevertheless, we should remain critical of this association since migraine is highly prevalent in females and since both diseases disproportionately affect this population.

\section{Declarations}

Conflict of interest None declared.

Informed consent The patient consented to the publication of this case report.

\section{References}

1. Elgendy IY, Nadeau SE, Bairey Merz CN, Pepine CJ, American College of Cardiology Cardiovascular Disease in Women Committeed, American College of Cardiology Cardiovascular Disease in Women C. Migraine headache: an under-appreciated risk factor for cardiovascular disease in women. J Am Heart Assoc. 2019;8(22):e014546.

2. Grosskopf J, Wohrmann S, Betz M, Heckmann JG. Takotsubo cardiomyopathy and migraine. Cephalalgia. 2013;33(4):285-6.

3. Jalan P, Dhakal L, Pandav V, Omar AI. Status migrainosus as a potential stressor leading to takotsubo cardiomyopathy. Cephalalgia. 2012;32(15):1140-3.

4. Finsterer J, Wahbi K. CNS disease triggering Takotsubo stress cardiomyopathy. Int J Cardiol. 2014;177(2):322-9.

5. Kline JA, Mitchell AM, Kabrhel C, Richman PB, Courtney DM. Clinical criteria to prevent unnecessary diagnostic testing in emergency department patients with suspected pulmonary embolism. J Thromb Haemost. 2004;2(8):1247-55.

6. Scantlebury DC, Prasad A, Rabinstein AA, Best PJ. Prevalence of migraine and Raynaud phenomenon in women with apical ballooning syndrome (Takotsubo or stress cardiomyopathy). Am J Cardiol. 2013;111(9):1284-8. 